University of Wollongong

Research Online

Faculty of Social Sciences - Papers (Archive) Faculty of Arts, Social Sciences \& Humanities

2015

Dementia attitudes and help-seeking intentions: an investigation of responses to two scenarios of an experience of the early signs of dementia

Lyn Phillipson

University of Wollongong, Iphillip@uow.edu.au

Christopher A. Magee

University of Wollongong, cmagee@uow.edu.au

Sandra C. Jones

University of Wollongong, Sandra.Jones@acu.edu.au

Samantha L. Reis

University of Wollongong, sreis@uow.edu.au

Ellen Skladzien

Alzheimer's Australia Inc, Ellen.Skladzien@alzheimers.org.au

Follow this and additional works at: https://ro.uow.edu.au/sspapers

Part of the Education Commons, and the Social and Behavioral Sciences Commons

Research Online is the open access institutional repository for the University of Wollongong. For further information contact the UOW Library: research-pubs@uow.edu.au 


\title{
Dementia attitudes and help-seeking intentions: an investigation of responses to two scenarios of an experience of the early signs of dementia
}

\begin{abstract}
Objectives: To investigate associations between dementia-attitudes and help-seeking intentions. Method: An online survey of 611 Australian adults (45-60 years) assessed dementia-related attitudes and helpseeking intentions in response to two scenarios of an experience of early dementia: for themselves (Scenario 1); and for a significant other (proxy help-seeking) (Scenario 2). Logistic regression models examined the relationship between four dementia-related attitudes (labelled Personal Avoidance, Fear of Labelling, Fear of Discrimination and Person Centredness) and help-seeking intentions. Results: Most participants indicated they would seek help from a general practitioner (GP) for themselves (82.2\%) or for a proxy $(78.7 \%)$ in response to the scenarios. Whilst only $7.2 \%$ indicated they would seek help from noone, $21.3 \%$ would delay seeking help. In response to Scenario 1, Personal Avoidance and Fear of Labelling were associated with intentions to delay help-seeking. Fear of both Labelling and Discrimination were associated with intentions to seek help from no-one. In response to Scenario 2, Personal Avoidance was associated with intentions to delay proxy help-seeking and a reduced likelihood of seeking help by phone or and with Fear of Discrimination, via a GP. Fear of Labelling was also associated with an intention to delay proxy help-seeking. Conclusion: Efforts to improve help-seeking for dementia should address attitudes relating to stigma including negative labelling and a desire for the avoidance of people with dementia. Fears relating to discrimination indicate a need to build public confidence regarding the capacity of the health and workforce sectors to support people with dementia ethically and appropriately.
\end{abstract}

\section{Keywords}

seeking, intentions, investigation, responses, two, help, scenarios, dementia, experience, early, signs, attitudes

\section{Disciplines}

Education | Social and Behavioral Sciences

\section{Publication Details}

Phillipson, L., Magee, C., Jones, S., Reis, S. \& Skladzien, E. (2015). Dementia attitudes and help-seeking intentions: an investigation of responses to two scenarios of an experience of the early signs of dementia. Aging and Mental Health, 19 (11), 968-977. 
"This article was originally published as" Dementia attitudes and help-seeking intentions: an investigation of responses to two scenarios of an experience of the early signs of dementia

Phillipson, L., Magee, C., Jones, S.C, Reis, S. and Skladzien, E. (2015).

Associations between help-seeking intentions and stigma in response to the early signs and symptoms of dementia, Aging and Mental Health, Aging and Mental Health

\section{Abstract}

Objectives: To investigate associations between dementia-attitudes and help-seeking intentions. Methods: An online survey of 611 Australian adults (45 - 60 years) assessed dementia related attitudes and help-seeking intentions in response to two scenarios of an experience of early dementia: for themselves (Scenario 1); and for a significant other (proxy help-seeking) (Scenario 2). Logistic regression models examined the relationship between four dementia-related attitudes (labelled Personal Avoidance, Fear of Labelling, Fear of Discrimination and Person Centredness) and help-seeking intentions. Results: Most participants indicated they would seek help from a GP for themselves $(82.2 \%)$ or for a proxy $(78.7 \%)$ in response to the scenarios. Whilst only $7.2 \%$ indicated they would seek help from no-one, $21.3 \%$ would delay seeking help. In response to Scenario 1, Personal Avoidance and Fear of Labelling were associated with intentions to delay help-seeking. Fear of both Labelling and Discrimination were associated with intentions to seek help from no-one. In response to Scenario 2, Personal Avoidance was associated with intentions to delay proxy help seeking and a reduced likelihood of seeking help by phone or and with Fear of Discrimination, via a GP. Fear of Labelling was also associated with an intention to delay proxy help-seeking. Conclusions: Efforts to improve help-seeking for dementia should address attitudes relating to stigma including negative labelling and a desire for the avoidance of people with dementia. Fears relating to discrimination indicate a need to build public confidence regarding the capacity of the health and workforce sectors to support people with dementia ethically and appropriately. 
Key words: Dementia, stigma, help-seeking, diagnosis 


\section{Introduction}

Dementia is a major and growing health and social concern that places considerable burdens on families, carers, and society in general (Lanctôt, Rajaram, \& Herrmann, 2009; Scarpini, Scheltens, \& Feldman, 2003). Many health professionals and community members believe that screening and early diagnosis of dementia is of little benefit given the lack of known cures (Terpening, Hodges, \& Cordato, 2011). Thus, many individuals with dementia and their families delay seeking help in the early stages of the condition (Benbow \& Jolley, 2012) contributing to the delays in diagnosis observed in both Europe and Australia (Speechly, Bridges-Webb, \& Passmore, 2008; Wilkinson, Sganga, Stave, \& O’Connell, 2005). However, early diagnosis can promote early access to medications that relieve symptoms (Farlow \& Cummings, 2007) and interventions that slow disease progression for certain types of dementia (e.g., vascular dementia) (Leifer, 2003; Terpening et al., 2011). In addition, early diagnoses can enable patients and family members to actively plan future care (Bradford, Kunik, Schulz, Williams, \& Singh, 2009; Leifer, 2003). This can allow individuals to live for a longer period of time at home which is often preferred by individuals and family caregivers (Eagar et al., 2007) and can minimise the strain on health and residential aged care systems (Chrisp, Tabberer, Thomas, \& Goddard, 2012).

Unfortunately, numerous barriers exist to the early detection and diagnosis of dementia (Phillips, Pond, \& Goode, 2011). These include system level factors such as a lack of accurate screening, limited system resources (Bradford et al., 2009), and a reluctance by general practitioners (GPs) to make a diagnosis of dementia (Bradford et al., 2009; Hansen, Hughes, Routley, \& Robinson, 2008). Early detection of dementia can also be inhibited by a reluctance of individuals to seek help if they notice early signs of dementia (Benbow \& Jolley, 2012); this may reflect attitudes, communication problems, and knowledge deficits 
among patients, families, and healthcare professionals regarding the efficacy of early interventions (Bradford et al., 2009).

\section{Dementia-related attitudes}

Attitudes reflecting components of stigma may also play an important role in impeding help seeking behaviours for dementia. For example, stigma may lead to delayed diagnosis (Bradford et al., 2009; Vernooij-Dassen et al., 2005), as observed with conditions such as depression, and infectious diseases such as HIV (Barney, Griffiths, Jorm, \& Christensen, 2006; Byrne, 2001; Fife \& Wright, 2000; Parker \& Aggleton, 2003). Stigma is a multifaceted concept and there is considerable variation in the way stigma has been defined and measured in the literature (Deacon, 2006; Link \& Phelan, 2006). Link \& Phelan (2001, 2006) proposed a model that conceptualises stigma as a process encompassing many concepts discussed in the broader literature such as labelling, stereotyping, separation, status loss, discrimination and the negative use of power (Link \& Phelan, 2001, 2006). These concepts are briefly outlined below, and discussed in relation to the current dementia literature.

Labelling is a socially driven process, whereby individuals have a tendency to identify and label human differences (e.g., the presence or absence of a disability). The use of labelling often leads to oversimplification and categorisation of individuals. In the present context, there is evidence that the labelling of people with Alzheimer's disease (AD) has led to a social construction of people with dementia which is particularly dehumanising. Such labels have identified people with dementia as in a state 'between life and death', with references to 'zombies' and 'the living dead' being applied to those with the condition (Aquilina \& Hughes, 2006; Behuniak, 2011b). Some have argued that much of this labelling has arisen from the dominance of the biomedical model to understand disease (Kitwood, 1997). 
Labelling can feed into the creation of Stereotypes, which link undesirable characteristics to groups of people (Link \& Phelan, 2001, 2006). Iliffe et al. (2005) noted that because dementia is a disease associated with ageing, it may be associated with (and feared because of) negative ageist stereotypes such as dependence, loss of autonomy and dignity, and the prospect of institutionalisation.

The third component of stigma involves a separation process of 'us from them' (Link $\&$ Phelan, 2001, 2006). For example, people with dementia are considered to 'feel differently from others' and are difficult to communicate with (Crisp, Gelder, Rix, Meltzer, \& Rowlands, 2000). This process can then lead to the fourth component of stigma: the experience of discrimination for the stigmatised group (Link \& Phelan, 2001, 2006). Individual discrimination can occur when labelling and stereotyping lead a person to treat the stereotyped group differently; this can include avoiding individuals, or more overt forms of discrimination. It appears that people with dementia and their carers experience these types of discrimination, which can contribute to social isolation, avoidance and exclusion (Katsuno, 2005). There is also evidence that some health professional and care staff display discriminatory behaviours such as infantilisation, invalidation, banishment, ignoring, withholding, mockery, and disparagement (Kitwood, 1997).

In the present context, discrimination (which includes a loss of status that limits the number of opportunities available to a group) can also result in an expectation of discriminatory behaviours or practises; this expectation has been termed stigma consciousness. Link and Phelan (2006) argue this insidious form of discrimination can lead to loss of status and the individual being devalued because they attract the 'label' of the disease. Being conscious of the label has the potential to influence social behaviour because of the expectation of stigma. Among people diagnosed with dementia this may express itself 
in experiences of internalised shame or an anticipation of social rejection (Burgener \& Berger, 2008)

Structural or secondary discrimination refers to institutional practises that work to disadvantage stereotyped groups (e.g.; funding allocation disparities for research or treatment) (Milne, 2010). As an illustration of comparative funding shortfalls associated with dementia, between 2002 and 2007, dementia research in Australia received only half the funding compared to cancer research (when weighted by disease burden). As a proportion of direct care costs, the extent of this funding shortfall is more pronounced, with dementia research receiving less than one- fifth of the funding allocated to cancer or diabetes (Alzheimer's Australia, 2011) suggesting that secondary discrimination for dementia exists in Australia at least in relation to funding disparities.

Finally, Link and Phelan (Link \& Phelan, 2001, 2006) propose that stigmatisation is dependent on power, which refers to social, economic, or political forces. Power is important as it influences the extent to which the components of stigma described above co-occur. Link and Phelan $(2001,2006)$ argue that stigmatisation usually occurs in a situation where one group (the stigmatised) has less power (due to their illness, disability, difference etc) allowing labelling, stereotyping, status loss, and discrimination to occur.

\section{The Present Study}

Whilst recent studies are continuing to build our understanding of experiences of stigma in early dementia (Burgener et al., 2013), there have only been limited investigations into the nature of the relationship between stigma-related attitudes towards dementia and help seeking behaviours when the early signs and symptoms are noticed. Such investigations are needed to inform comprehensive interventions to reduce stigma that will also lesson any related obstacles to help-seeking (e.g. Gulliver, 2010). 
To address this gap, the present study examined whether the stigma-related attitudes associated with social exclusion (including labelling, a desire for personal avoidance and discriminatory attitudes) (Phillipson, Magee, Jones, \& Skladzien, 2012), were associated with help-seeking intentions in response to two 'hypothetical' experiences of the early signs and symptoms of dementia in middle aged adults. In addition, we also examined the relationship between more person-centred, positive attitudes towards people with dementia. This was an important consideration given the growing emphasis on the need to promote more person centred views of people living with dementia (Kitwood, 1997) and was also important to contribute to a more balanced discourse around stigma that acknowledged the potential for positive perceptions to contribute to positive help-seeking behaviours.

The study examined both intentions to help-seek for one-self and intentions to helpseek on behalf of someone else (a family member or loved one). The latter was included since help seeking for dementia is often initiated by a close family member (e.g., a husband or wife).

The study of hypothetical real-life situations was considered useful to explore the degree to which aspects of dementia-related stigma had affected a general adult population, most of whom were yet to experience any signs or symptoms of dementia. Targeting 'middle -aged' adults is of significant interest as targets for potential stigma reduction interventions as they are, in many circumstances, proxy help-seekers (i.e., those who seek help on behalf of the individual with dementia); including both actual (e.g. for their aged parents) and potential future proxy help-seekers (e.g. for spouse or other family members). Finally, without advancements in treatment or cures, they will also be the next generation who will experience dementia themselves in significant numbers (Ferri et al., 2005).

\section{Methods}




\section{Participants}

Participants were middle aged Australian adults aged 40 - 65 years old, recruited via targeted advertisements placed on the Facebook pages of users from this age segment and purchased on a per click basis. Links to the online survey were also distributed nationally via both the 'National Seniors' newsletters, and through Alzheimer's Australia national database of email and via their national newsletters. Participants were excluded if they reported having received a medical diagnosis of dementia, but not if they 'felt concerned they may have some of the early signs or symptoms of dementia'. This was to be inclusive of those who may have mild cognitive impairment, but not yet a formal diagnosis of dementia. Informed consent was obtained from all participants and ethical approval for this study was obtained from our university's Human Research Ethics Committee.

\section{Measures}

Participants completed an online questionnaire assessing social and demographic characteristics, dementia stigma and help seeking intentions.

Information on age, sex, country of birth (Australia, other country), education level ( $\leq$ High School, technical college, University), marital status (single/divorced, married/defacto), employment status (not working, casual/part-time, full-time), and household income $(\leq$ AUD $\$ 50,000, \$ 50,001-\$ 100,000, \geq \$ 100,001)$ were collected through the questionnaire. Participants indicated whether they had a confirmed diagnosis of dementia (yes versus no); whether they were they concerned that they themselves had early signs of dementia (yes versus no); and whether they had ever been a primary carer for someone with Alzheimer's Disease or another type of Dementia (yes versus no). 
Stigma and attitudes relating to dementia were examined using a 31-item questionnaire (Phillipson et al., 2012). This included 12 items from the Perceptions Regarding Investigational Screening for Memory in Primary Care (PRISM-PC) - Dementia Screening Subscale (Boustani et al., 2008) which assesses hypothetical responses to a diagnosis of dementia (e.g., 'I would feel humiliated'). Nine items were adapted from the Fraboni scale of Ageism (Fraboni, Saltstone, \& Hughes, 1990), and assessed attitudes relating to a desire for separation and avoidance from people with dementia (e.g., 'It is best that people with dementia live where they won't bother anyone'). Ten items were also developed in consultation with the Alzheimer's Australia Consumer Dementia Research Network (Alzheimer's Australia, 2010) to assess person centred/positive views of individuals living with dementia (e.g., 'People with dementia are a good source of knowledge' and 'People with dementia are respected for their wisdom'). All items were assessed on a 5-point Likert-type response format ('strongly agree' to 'strongly disagree').

Exploratory factor analysis (EFA) and confirmatory factor analysis (CFA) were performed on random halves of the sample to examine the presence of distinct attitudinal factors. For the EFA, the number of factors was determined using Velicer's Minimum Average Partial Test (O'Connor, 2000), which indicated four distinct factors. These four factors were then extracted using exploratory factor analysis conducted using direct oblimin rotation. Three items had very low factor loadings, but were retained in the CFA. The CFA, conducted with Mplus version 6.11 (Muthén \& Muthén, 1998 - 2010), then examined whether these four factors provided an adequate fit of the data. Model fit was assessed by indices such as root mean square error of approximation (RMSEA), standardized root mean square residual (SRMR), comparative fit index (CFI) and Tucker-Lewis Index (TLI) (Hu \& Bentler, 1998). Although the RMSEA (.05) and SRMR (.08) were within acceptable ranges, the CFI (.89) and TLI (.88) were a little low (Hu \& Bentler, 1998). As with the EFA, the 
loadings for three items were low. When these items were removed from the model, the model fit improved significantly, $\chi^{2} 84=216.06, p<.001$; there were also improvements in the RMSEA (.05), SRMR (.06), TLI (.92), and CFI (.91).

The EFA and CFA therefore suggested four distinct attitudinal factors. The Personal Avoidance factor (8 items; Cronbach's $\alpha=.84$ ) assessed social exclusion/avoidance of individuals with dementia (e.g. 'It is best that people with dementia live where they won't bother anyone'). The Person Centeredness factor (8 items; Cronbach's $\alpha=.76$ ) assessed positive views of individuals living with dementia (e.g., 'People with dementia pass on valued traditions'). Fear of Labelling (8 items; Cronbach's $\alpha=.86$ ) assessed participant responses to a hypothetical diagnosis of dementia (e.g., 'I would feel humiliated'). Fear of Discrimination (4 items; Cronbach's $\alpha=.76$ ) assessed concerns that the individual would not receive adequate care if they had dementia, and fears associated with Health Insurance Company and employers finding out about their diagnosis.

\section{[INSERT FIGURE 1]}

Help-seeking intentions were assessed using the Help Seeking Scale (Wilson, Deane, Ciarrochi, \& Rickwood, 2005) to explore the likelihood that participants would seek help from a partner, friend, parent, other family member, counsellor/psychologist, phone help line, or a doctor/GP if they were concerned they had early signs of dementia (e.g., memory loss) (Scenario 1). Two items also assessed whether participants would not seek help from anyone or delay seeking help for as long as possible if they thought they had the early signs of dementia. All items were assessed on a 5-point Likert-type response format ('extremely likely' to 'extremely unlikely'); an additional 'not applicable' option was also provided where appropriate as the age of this sample may mean that many individuals do not have living parents or a partner. The Help Seeking Scale was also utilised to test whether 
participants would seek help on behalf of a friend or family member who was showing early signs of dementia (Proxy Help-seeking) (Scenario 2). For the purposes of this paper, responses to the Help Seeking Scale items were merged into three categories: likely (extremely likely and likely); unlikely (extremely unlikely and unlikely); and other (neither likely or unlikely, and not applicable).

\section{Statistical Analysis}

The relationship between the attitudinal domains and help seeking intentions for dementia were examined using multinomial logistic regression conducted with IBM SPSS Statistics for Windows Version 19 (IBM Corporation, New York). This involved examining the relationships between the attitudinal factors and help-seeking intentions. Separate models were tested for each help-seeking item (e.g., intentions to seek help from a partner), with each model including all four attitudinal factors entered simultaneously. The models specified 'unlikely' as the referent category, and we reported the odds ratios (with 95\% confidence intervals) for the 'likely' versus 'unlikely' comparison (for reasons of space, 'other' versus 'unlikely' results are not reported). The following covariates were controlled for in each model: age, sex, country of birth, marital status, education level, source of recruitment, and dementia carer status.

Interaction terms were added separately to each model to examine whether the relationships of each attitudinal factor with help seeking intentions varied by carer status, experience of early signs of dementia, age, and sex. The significance of each interaction term was determined using likelihood ratio tests, and a conservative $\mathrm{p}$ value of .01.

\section{Results}


There were 619 responses to the online survey. Eight respondents indicates that they had a diagnosis of dementia so were excluded from the study, leaving the final sample of 611 adults aged $40-65$ years $(M=54.4 ; S D=17.0), 78.2 \%$ of whom were female $(n=482)$ and $21.8 \%$ were male $(n=134)$. Approximately one-third of participants $(n=183 ; 30 \%)$ indicated they were concerned they were experiencing the early signs and symptoms of dementia, but did not have a diagnosis of dementia. One third of the sample also indicated they had been a primary carer for someone with dementia, and $89.9 \%$ knew someone who had dementia. Participants were recruited via a number of sources including Facebook advertisements (39.9\%), newsletters and emails distributed by National Seniors - a not-for-profit Australian organisation supporting individuals aged over 50 years $(27.7 \%)$, and emails distributed by Alzhiemers Australia (15.9\%). The remaining 16.5\% of participants received emails about the study through friends, family members, or work colleagues recruited through the above sources. Other demographic characteristics of the sample are presented in Table 1.

[Insert Table 1]

\section{Early Help Seeking Intentions and Dementia Attitudes in Australian Adults}

Scenario 1: help-seeking intentions for self for participants in response to the early signs of dementia

Figure 2 outlines the responses to the help seeking items in response to Scenario 1: that the respondent themselves was experiencing the early signs of dementia.

\section{[Insert Figure 2]}

The majority of respondents indicated they would seek help if they thought they had the early signs and symptoms of dementia and were most likely to do so from a GP $(82.2 \%)$ or a partner (49.4\%). Whilst very few people indicated that they would not seek help from 
anyone if they thought they had the early signs of dementia (7.2\%), more than one-fifth of the sample $(21.3 \%)$ indicated that they would delay seeking help for as long as possible.

[Insert Table 2]

Table 2 outlines the associations between the help-seeking items for participants in response to Scenario 1. As shown in Table 3, Person Centredness was not significantly associated with help seeking intentions from any source. Personal Avoidance was significantly associated with an increased likelihood of delaying help seeking for as long as possible $(\mathrm{OR}=1.07,95 \%$ confidence interval $[1.01,1.13])$.

Fear of Labelling was associated with an increased likelihood of not seeking help from anyone $(\mathrm{OR}=1.09[1.02,1.16])$ and an increased likelihood of delaying help seeking for as long as possible $(\mathrm{OR}=1.08[1.04,1.13])$.

Fear of Discrimination was significantly associated with a reduced likelihood of seeking help from a psychologist or counsellor $(\mathrm{OR}=.89,[.82, .95])$. Fear of Discrimination was also associated with a reduced likelihood of seeking help from a general practitioner (OR $=.81[.72, .90])$. Fear of Discrimination was also found to be significantly associated with an increased likelihood of not seeking help from anyone $(\mathrm{OR}=1.14[1.02,1.28])$.

The results of the interaction terms indicated that most of the relationships between attitudinal factors and help seeking intentions did not vary significantly by age, sex, carer status, and early signs of dementia. However, the relationship between Fear of Labelling and seeking help from a friend was found to differ significantly between individuals who reported noticing they had early signs of dementia compared with those who did not $\left(\chi_{2}^{2}\right.$ for difference $=9.24, p=.010)$. Further inspection of this effect indicated that Fear of Labelling 
was significantly associated with a reduced likelihood of help seeking from a friend in individuals reporting having early signs of dementia $(\mathrm{OR}=.90,[.83, .99])$ but not in those who did not report having early signs of dementia $(\mathrm{OR}=.99[.95,1.04])$.

The relationship between Fear of Discrimination and help seeking intentions from a general practitioner differed significantly between individuals who did and did not report having the early signs of dementia $\left(\chi_{2}^{2}\right.$ for difference $\left.=10.08, p=.006\right)$. Further investigation of this effect indicated that Fear of Discrimination was associated with a reduced likelihood of seeking help from a general practitioner only in individuals who did not report having the early signs of dementia $(\mathrm{OR}=.68[.59, .80])$.

Scenario 2: help-seeking intentions for a significant other (proxy help-seeking) in response to the early signs of dementia

\section{[Insert Table 3]}

Table 3 outlines the help seeking intentions of participants in relation to Scenario 2: seeking help on behalf of someone else (proxy help-seeking). Participants were more likely to indicate that they would seek help on behalf of someone else (as opposed to for themselves) if they felt that individual had the early signs of dementia. Very few individuals indicated that they would not seek help from anyone $(3.8 \%)$ or that they would delay diagnosis for as long as possible (11.1\%). In terms of sources of assistance, participants who indicated that they would seek help on behalf of someone else were most likely to indicate they would do so from a GP (78.7\%), followed by a psychologist (55.8\%), a phone counsellor $(52.9 \%)$ or a relative $(50.9 \%)$.

In regards to the attitudinal profile of proxy help-seekers, Personal Avoidance was linked with a reduced likelihood to seek help over the phone (OR $=.92[.87, .97])$. Personal 
Avoidance was associated with a reduced likelihood to seek help from a GP (OR $=.92[.86$, .98]). Personal Avoidance was associated with an increased likelihood of delaying help seeking for as long as possible $(\mathrm{OR}=1.07[1.00,1.14])$.

Person Centredness was not associated with any help seeking intentions. However, Fear of labelling was also significantly associated with an increased likelihood of delaying help seeking $(\mathrm{OR}=1.06[1.00,1.12])$. Fear of Discrimination was linked with a reduced likelihood of seeking help from a GP $(\mathrm{OR}=.90[.81,1.00])$.

The association between Personal Avoidance and delaying help seeking differed significantly by $\operatorname{sex}\left(\chi^{2} 2\right.$ for difference $\left.=15.68, \mathrm{p}<.001\right)$. Further inspection of this interaction indicated that a significant association between Personal Avoidance and delaying help seeking was observed in males $(\mathrm{OR}=1.54[1.21,1.95])$ but not females $(\mathrm{OR}=1.00$ $[.92,1.08])$. None of the other interaction terms were statistically significant.

\section{Discussion}

The present study explored the association between dementia attitudes and the helpseeking intentions of Australian adults in response to two scenarios: that they themselves had the early signs of dementia (Scenario 1) or that a significant other was experiencing the early signs (Scenario 2). Our findings indicate that the majority of respondents would seek help either for themselves, or a loved one, if there were concerns they may be experiencing the early signs of dementia. Preferred sources of help-seeking for self indicate a strong orientation towards both professionals, primarily but not exclusively GPs, as well as personal channels (especially partners). These results are consistent with the current literature regarding the importance of GPs in the primary care setting in diagnosing dementia (Iliffe et al 2005) but also underscore the important role of other services such as psychologists and 
helplines, along with the need for family and spousal in supporting early help-seeking behaviours for possible dementia.

If GPs are to effectively play this important role as primary and preferred source for early help-seekers there is evidence of a need for improved education and capacity building within primary care. This is especially as it relates to their skills to provide an accurate diagnosis and the need to adopt a less fatalistic approach to early dementia diagnosis, (Phillips et al 2011, Boustani et al 2008). If family members and partners are also to play this critical support role and encourage early help-seeking there also appears to be a need for further public education regarding the associated benefits of early help-seeking in dementia and pathways to diagnosis, support and care (Iliffe et al. 2005; Speechly et al. 2008).

Whilst only a small proportion (7.2\%) of respondents indicated that they would seek help-from 'no-one' in response to the 'early signs of dementia', a greater proportion $(21.3 \%)$ indicated they would 'delay help-seeking for as long as possible'. Overall, the results suggest that certain elements of stigma were significantly associated with reduced help seeking intentions in the scenario presented to participants. Attitudes associated with a desire for 'Personal Avoidance' of people with dementia, and fears relating to 'Labelling' and 'Discrimination' associated with dementia (e.g., 'I would not want my family to know'; 'I would feel humiliated') were significantly linked with intentions to either delay or avoid seeking help for the early signs dementia. Higher fear of 'Discrimination' was also associated with lower intentions to seek help from either a GP or a psychologist.

Results also indicated that some associations varied significantly depending on whether individuals reported experiencing early signs of dementia. For instance, an association between Fear of Labelling and a reduced likelihood of seeking help from friends was observed in individuals who reported having early signs of dementia. Interestingly, Fear 
of Discrimination was associated with a reduced likelihood of seeking help from a GP only in individuals who did not report having early signs of dementia. A desire for 'Personal Avoidance', and a 'Fear of Labelling' were also significantly associated with intentions to delay proxy help-seeking. Fear of Discrimination was also associated with lower intentions of proxy help-seeking from a GP. Interestingly, the association between a desire for Personal Avoidance of people with dementia and delaying proxy help-seeking was evident in males but not in females, suggesting a particular need for strategies specifically focused on supporting male help-seeking through targeted education and additional support.

\section{Strengths and Limitations}

The present paper is limited by several factors including the cross-sectional design, convenience sampling which limits the generalizability of results, and the focus on helpseeking intentions, which may not necessarily translate into behaviour. Furthermore, the vast majority of participants indicated that they knew someone with dementia, which may have influenced the responses to the items in this question. It is possible that individuals with less experience of dementia may have a different pattern of responding, which requires investigation. In aggregate, this suggests a need for more research, particularly involving longitudinal designs, to further clarify the nature of the relationship between attitudes relating to stigma and help seeking for dementia.

Despite these issues, the present results provide an important and timely insight into the nature of the relationships between attitudes about dementia, especially those related to aspects of stigma and the intentions of the public to help-seek for dementia. In particular, although negative and dehumanising labelling, stereotypes and discriminatory behaviours have been previously identified within the dementia literature (e.g. Aquilina \& Hughes 2006), this is the first study to identify a fear of both labelling and discrimination with the intentions 
of members of the general adult public to delay or avoid help-seeking for dementia for themselves, or on behalf of another, if they were experiencing the early signs and symptoms. These results, although preliminary, have a number of implications which are briefly discussed below.

\section{Implications}

Whilst such results may indicate a need for public awareness campaigns to promote more positive attitudes towards people with dementia (Benbow \& Jolley, 2012), the lack of association between 'person centred' attitudes and help-seeking intentions suggests the need for further reflection. Issues surrounding help seeking and early detection are somewhat controversial given that there are no cures for dementia. Some argue that early detection has few benefits and may have negative consequences such as distress and suicide ideation (Draper, Peisah, Snowdon, \& Brodaty, 2010). However, others have highlighted perceived benefits of early detection and diagnosis including individuals participating in future planning and access to pharmacological and non-pharmacological treatments available which can slow disease progression and extend quality of life (Leifer, 2003; Terpening et al., 2011). In this light, the present findings have important implications for improving the early detection of dementia. For example, although many system factors (e.g., GP time constraints) may impede the early detection of dementia (Bradford et al., 2009), domains of stigma may also impede help seeking. That is, it appears that the prospect of being discriminated against or being labelled as having the disease inhibits help seeking intentions.

Combating dementia-related stigma within primary care services may reduce the reluctance of some patients to express concerns regarding memory loss. Research into the effect of creating 'dementia friendly' general practices is required to assess whether altering perceptions towards this illness in a health care setting impacts on patients' willingness to 
seek help. As society ages, there will be a need to ensure a health workforce that is equipped to take a holistic approach to the care and treatment of people with dementia (Benbow \& Jolley, 2012). Negative labelling of dementia by health professionals has been linked to the dominance of the biomedical model (Kitwood, 1997) and beliefs that individuals with dementia are a 'non-person whose brain has been destroyed by the disease and who therefore no longer exists as a person, but only a body to be managed' (Behuniak, 2011a). Such critique calls for a new approach to the education of health professionals oriented towards person-centred care where the emphasis is placed on the person and not on the symptoms of the disease (Kitwood 1997).

At the community level, stigma could feasibly be addressed by improving awareness and knowledge about dementia including the benefits of early diagnosis (Benbow \& Jolley, 2012). Relevant messages could include emphasising that the progression of dementia is slow, that treatments are available, and that individuals are able to maintain their quality of life for a considerable amount of time (Benbow \& Jolley, 2012; Draper et al., 2010). Given the expressed fear of discrimination within the health sector, strategies may also be needed to improve public confidence that workers within the health sector will not discriminate against people with dementia but work to promote human rights and dignity in the healthcare system (Benbow \& Jolley 2012).

\section{Conclusions}

While the results should be verified in future studies, they have a number of implications for strategies to improve help seeking behaviours. Those discussed within this paper could play an important role to reduce dementia-related stigma and aid efforts to detect dementia early, slow the rate of disease progression, and enable the individual and family members to better plan for their future. 


\section{References}

Alzheimer's Australia. (2010). Consumer Involvement in Dementia Research:Alzheimer's Australia's Consumer Dementia Research Network. A report for Alzheimer's Australia (Vol. Paper 22).

Alzheimer's Australia. (2011). Budget Submission. Canberra, Australia.

Aquilina, C., \& Hughes, J. (2006). The return of the living dead: agency lost and found? In J. Hughes, Louw, S. J. and Sabat, SR (Ed.), Dementia : Mind, Meaning, and the Person (pp. 143-161.). Oxford, UK: Oxford University Press.

Barney, L., Griffiths, K., Jorm, A., \& Christensen, H. (2006). Stigma about depression and its impact on help-seeking intentions. Australian and New Zealand Journal of Psychiatry, 40(1), 51 - 54.

Behuniak, S. (2011a). The living dead? The construction of people with Alzheimer's disease as zombies. Ageing and Society, 31, 70-92. doi: 10.1017/s0144686x10000693

Behuniak, S. (2011b). The living dead? The construction of people with Alzheimer's disease as zombies. Ageing \& Society, 31, 70-92. doi: 10.1017/s0144686x10000693

Benbow, S. M., \& Jolley, D. (2012). Dementia: stigma and its effects. Neurodegenerative Disease Management, 2, 165 - 172.

Boustani, M., Perkins, A. J., Monahan, P., Fox, C., Watson, L., Hopkins, J., . . . Hendrie, H. C. (2008). Measuring primary care patients' attitudes about dementia screening. International Journal of Geriatric Psychiatry, 23(8), 812-820.

Bradford, A., Kunik, M. E., Schulz, P., Williams, S. P., \& Singh, H. (2009). Missed and delayed diagnosis of dementia in primary care: prevalence and contributing factors. Alzheimer Dis Assoc Disord, 23(4), 306-314. doi: doi: 10.1097/WAD.0b013e3181a6bebc.

Burgener, S., Buckwalter, K., Perkhounkova, Y., Liu, M., Riley, R., Einhorn, C., . . HahnSwanson, C. (2013). Perceived stigma in persons with early-stage dementia: Longitudinal findings: Part 1. Dementia. doi: 10.1177/1471301213508399

Burgener, S. C., \& Berger, B. (2008). Measuring perceived stigma in persons with progressive neurological disease: Alzheimer's dementia and Parkinson's Disease. [References]: Dementia: The International Journal of Social Research and Practice. Vol.7(1), Feb 2008, pp. 31-53.

Byrne, P. (2001). Psychiatric stigma. The British Journal of Psychiatry, 178(3), 281-284. doi: 10.1192/bjp.178.3.281

Chrisp, T. A., Tabberer, S., Thomas, B. D., \& Goddard, W. A. (2012). Dementia early diagnosis: triggers, supports and constraints affecting the decision to engage with the health care system. Aging Mental Health, 16(5), 559-565. doi: doi: 10.1080/13607863.2011.651794. Epub 2012 Feb 24.

Crisp, A. H., Gelder, M. G., Rix, S., Meltzer, H. I., \& Rowlands, O. J. (2000). Stigmatisation of people with mental illnesses. British Journal of Psychiatry, 177, 4-7.

Deacon, H. (2006). Towards a sustainable theory of health-related stigma: lessons from the HIV/AIDS literature. Journal of Community \& Applied Social Psychology, 16(6), 418-425. doi: 10.1002/casp.900

Draper, B., Peisah, C., Snowdon, J., \& Brodaty, H. (2010). Early dementia diagnosis and the risk of suicide and euthanasia. Alzheimer's \& Dementia, 6(1), 75-82. doi: http://dx.doi.org/10.1016/j.jalz.2009.04.1229 
Eagar, K., Owen, A., Williams, K., Westera, A., Marosszeky, N., England, R., \& Morris, D. (2007). Effective Caring: a synthesis of the international evidence on carer needs and interventions: Centre for Health Service Development, University of Wollongong.

Farlow, M. R., \& Cummings, J. L. (2007). Effective pharmacologic management of Alzheimer's disease. American Journal of Medicine, 120(5), 388-397.

Ferri, C. P., Prince, M., Brayne, C., Brodaty, H., Fratiglioni, L., Ganguli, M., . . Scazufca, M. (2005). Global prevalence of dementia: a Delphi consensus study. The Lancet, 366(9503), 2112.

Fife, B. L., \& Wright, E. R. (2000). The Dimensionality of Stigma: A Comparison of Its Impact on the Self of Persons with HIV/AIDS and Cancer. Journal of Health and Social Behavior, 41(1), 50-67.

Fraboni, M., Saltstone, R., \& Hughes, S. (1990). The Fraboni Scale of Ageism (FSA): An attempt at a more precise measure of ageism. Canadian Journal on Aging, 9, 56-66.

Gulliver, A. G., K.M., and Christensen, H. (2010). Perceived barriers and facilitators to mental health help-seeking in young people: a systematic review. BMC Psychiatry, 10(113). doi: doi:10.1186/1471-244X-10-113

Hansen, E. C., Hughes, C., Routley, G., \& Robinson, A. L. (2008). General practitioners' experiences and understandings of diagnosing dementia: Factors impacting on early diagnosis. Social Science \& Medicine, 67(11), 1776-1783.

Hu, L.-t., \& Bentler, P. M. (1998). Fit Indices in Covariance Structure Modeling: Sensitivity to Underparameterized Model Misspecification. Psychological Methods, 3, 424-453.

Iliffe, S., De Lepeleire, J., Van Hout, H., Kenny, G., Lewis, A., \& Vernooij-Dassen, M. (2005). Understanding obstacles to the recognition of and response to dementia in different European countries: A modified focus group approach using multinational, multi-disciplinary expert groups. Aging \& Mental Health, 9(1), 1-6.

Katsuno, T. (2005). Dementia from the inside: how people with early stage dementia evaluate their quality of life. Ageing and Society, 25, 197-214.

Kitwood, T. (1997). Dementia Reconsidered: the Person Comes First. Buckingham, UK: Open University Press.

Lanctôt, K. L., Rajaram, R. D., \& Herrmann, N. (2009). Review: Therapy for Alzheimer's disease: how effective are current treatments? Therapeutic Advances in Neurological Disorders, 2(3), 163-180. doi: 10.1177/1756285609102724

Leifer, B. P. (2003). Early diagnosis of Alzheimer's disease: clinical and economic benefits. Journal of the American Geriatriac Society, 51(5 Suppl Dementia), S281-288.

Link, B., \& Phelan, J. (2001). Conceptualising Stigma. Annual Review of Sociology, 27, 363385.

Link, B., \& Phelan, J. (2006). Stigma and its public health implications. The Lancet, 367(9509), 528-529.

Milne, A. (2010). The 'D' word: reflections on the relationship between stigma, discrimination and dementia. Journal of Mental Health, 19(3), 227-233.

Muthén, L. K., \& Muthén, B. O. (1998 - 2010). Mplus Users Guide. Sixth Edition. Los Angeles: CA: Muthén \& Muthén.

Parker, R., \& Aggleton, P. (2003). HIV and AIDS-related stigma and discrimination: a conceptual framework and implications for action. Social Science AND Medicine, 57(1), 13-24.

Phillips, J., Pond, P., \& Goode, S. (2011). Timely Diagnosis of Dementia:Can we do better? A report for Alzheimer's Australia (Vol. Paper 24). Canberra, Australia: Alzheimer's Australia. 
Phillipson, L., Magee, C., Jones, S. C., \& Skladzien, E. (2012). Correlates of Dementia Attitudes in a sample of middle-aged Australian adults. Australasian Journal on Ageing. doi: DOI: 10.1111/j.1741-6612.2012.00624.x

Scarpini, E., Scheltens, P., \& Feldman, H. (2003). Treatment of Alzheimer's disease: current status and new perspectives. The Lancet, Neurology, 2, 539-547

Speechly, C., Bridges-Webb, C., \& Passmore, E. (2008). The pathway to dementia diagnosis. Medical Journal of Australia, 189, 487-489.

Terpening, Z., Hodges, J. R., \& Cordato, N. J. (2011). Towards evidence-based dementia screening in Australia [editorial]. ; . Medical Journal of Australia, 194, 60-61.

Vernooij-Dassen, M. J., Moniz-Cook, E. D., Woods, R. T., De Lepeleire, J., Leuschner, A., Zanetti, O., . . Iliffe, S. (2005). Factors affecting timely recognition and diagnosis of dementia across Europe: From awareness to stigma. International Journal of Geriatric Psychiatry, 20(4), 377-386.

Wilkinson, D., Sganga, A., Stave, C., \& O’Connell, B. (2005). Implications of the Facing Dementia. Survey for health care professionals across Europe. International Journal of Clinical Practice, 59(146), 27-31.

Wilson, C., Deane, F., Ciarrochi, J., \& Rickwood, D. (2005). Measuring Help-Seeking Intentions: properties of the General Help-Seeking Questionnaire. Canadian Journal of Counselling, 39, 15 - 28. 
Table 1. Demographic characteristics of the sample of Australian adults included in this study.
$\mathrm{N}$
$\%$

Sex

Male

131

21.4

Female

480

78.6

Age

$40-49$ years

159

26.0

$50-54$ years

123

20.1

$55-59$ years

151

24.7

$\geq 60$ years

178

29.1

Country of Birth

Australia

466

76.3

Other country

145

23.7

Experience early signs

Yes

183

30.0

No

428

70.0

Carer

Yes

183

30.0

No

428

70.0

Know someone

Yes

554

89.9

No

62

10.1

Source of recruitment

Facebook

244

39.9

National seniors

169

27.7

AA email

97

15.9 


\begin{tabular}{|c|c|c|}
\hline Other & 101 & 16.5 \\
\hline \multicolumn{3}{|l|}{ Marital Status } \\
\hline Single & 228 & 37.3 \\
\hline Partnered & 383 & 62.7 \\
\hline \multicolumn{3}{|l|}{ Education level } \\
\hline$\leq$ High School & 128 & 20.9 \\
\hline Trade/diploma/certificate & 179 & 29.3 \\
\hline University & 304 & 49.8 \\
\hline \multicolumn{3}{|l|}{ Employment status } \\
\hline Not working & 216 & 35.4 \\
\hline Casual/part-time & 197 & 32.2 \\
\hline Full-time & 198 & 32.4 \\
\hline \multicolumn{3}{|l|}{ Household income (annual) } \\
\hline$\leq A U D 50,000$ & 215 & 35.2 \\
\hline AUD 50,000 - 100,000 & 256 & 41.9 \\
\hline$>A U D 100,000$ & 140 & 22.9 \\
\hline
\end{tabular}


Table 2. Relationship between dementia attitudes and likelihood of seeking help from different sources for self. Results are reported as odds ratios.

\begin{tabular}{|c|c|c|c|c|c|c|c|c|c|}
\hline & Partner & Friend & Parent & Relative & $\begin{array}{c}\text { Psychologi } \\
\text { st }\end{array}$ & Phone & GP & No-one & Delay \\
\hline Person Centredness & 1.02 & .99 & .96 & .96 & 1.00 & .99 & .99 & 1.06 & 1.05 \\
\hline Fear of Labelling & .96 & .97 & .98 & .97 & .99 & .97 & .97 & $1.09 *$ & $1.08 *$ \\
\hline Fear of Discrimination & 1.00 & .97 & .95 & .93 & $.89 * *$ & 1.03 & $.81 * *$ & $1.14^{*}$ & 1.07 \\
\hline Nagelkerke $\mathrm{R}^{2}$ & .38 & .09 & .15 & .14 & .10 & .09 & .15 & .17 & .17 \\
\hline
\end{tabular}

Covariates included: Age, gender, marital status, education level, country of birth, recruitment source, and carer status.

$* \mathrm{p}<.05$

$* * \mathrm{p}<.01$ 
Table 3. Relationship between dementia attitudes and likelihood of seeking help from different sources on behalf of others. Results are reported as odds ratios.

\begin{tabular}{|c|c|c|c|c|c|c|c|c|}
\hline & Friend & Parent & Relative & Psychologist & Phone & GP & No-one & Delay \\
\hline Personal Avoidance & .99 & 1.05 & .98 & .98 & $.92 *$ & $.92 *$ & 1.03 & $1.07 *$ \\
\hline Person Centredness & 1.01 & 1.00 & 1.01 & 1.01 & 1.00 & 1.01 & 1.00 & 1.00 \\
\hline Fear of Labelling & .99 & .99 & 1.00 & 1.01 & 1.00 & .98 & 1.03 & $1.06 *$ \\
\hline Fear of Discrimination & 1.00 & .97 & .98 & .95 & 1.03 & $.90 *$ & 1.17 & 1.08 \\
\hline Nagelkerke $\mathrm{R}^{2}$ & .08 & .19 & .07 & .12 & .10 & .12 & .15 & .13 \\
\hline
\end{tabular}

Covariates included: Age, gender, marital status, education level, country of birth, recruitment source, and carer status.

$* \mathrm{p}<.05$

$* * \mathrm{p}<.01$ 


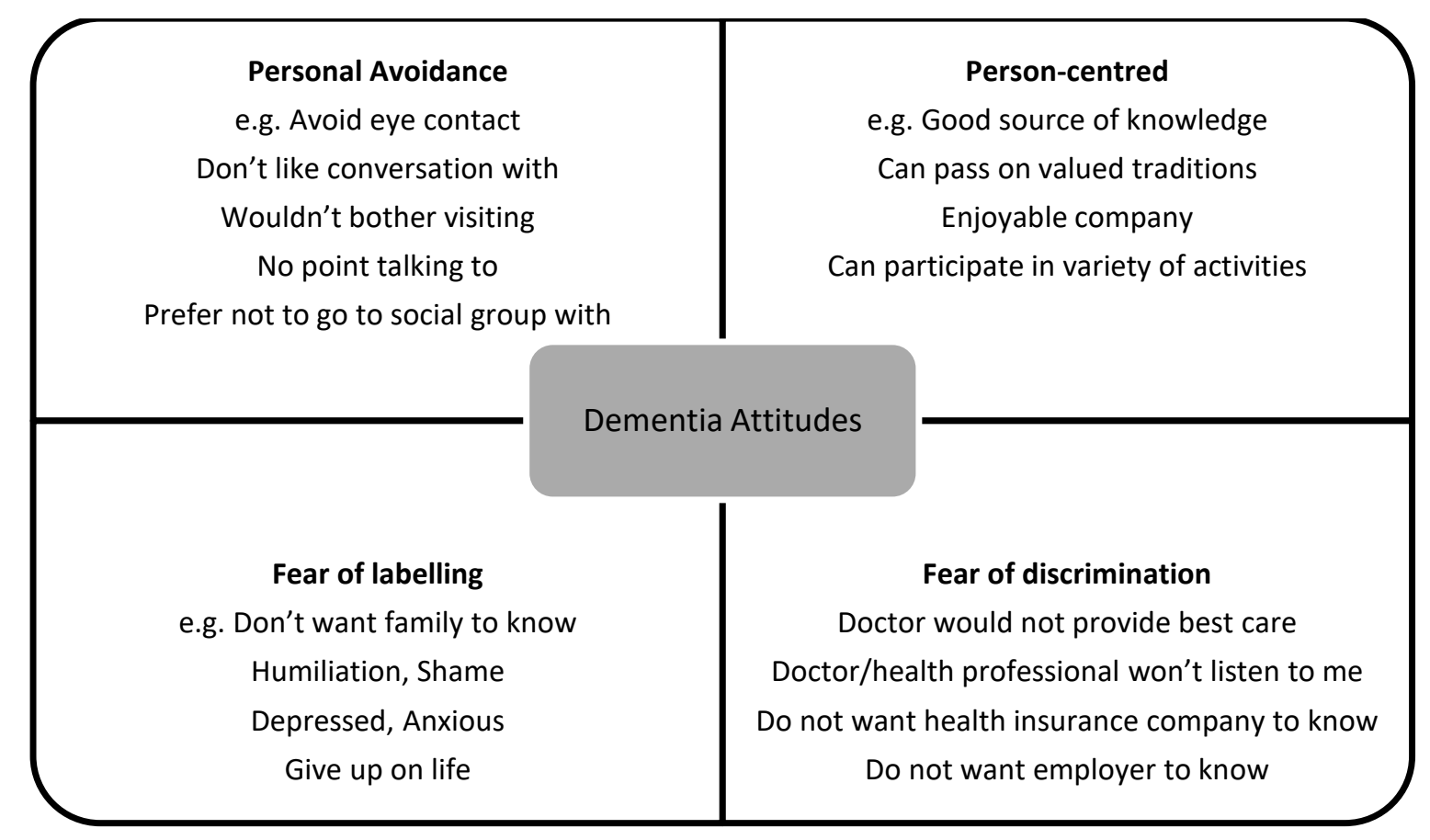

Figure 1 - The four factors indicating dementia attitudes 


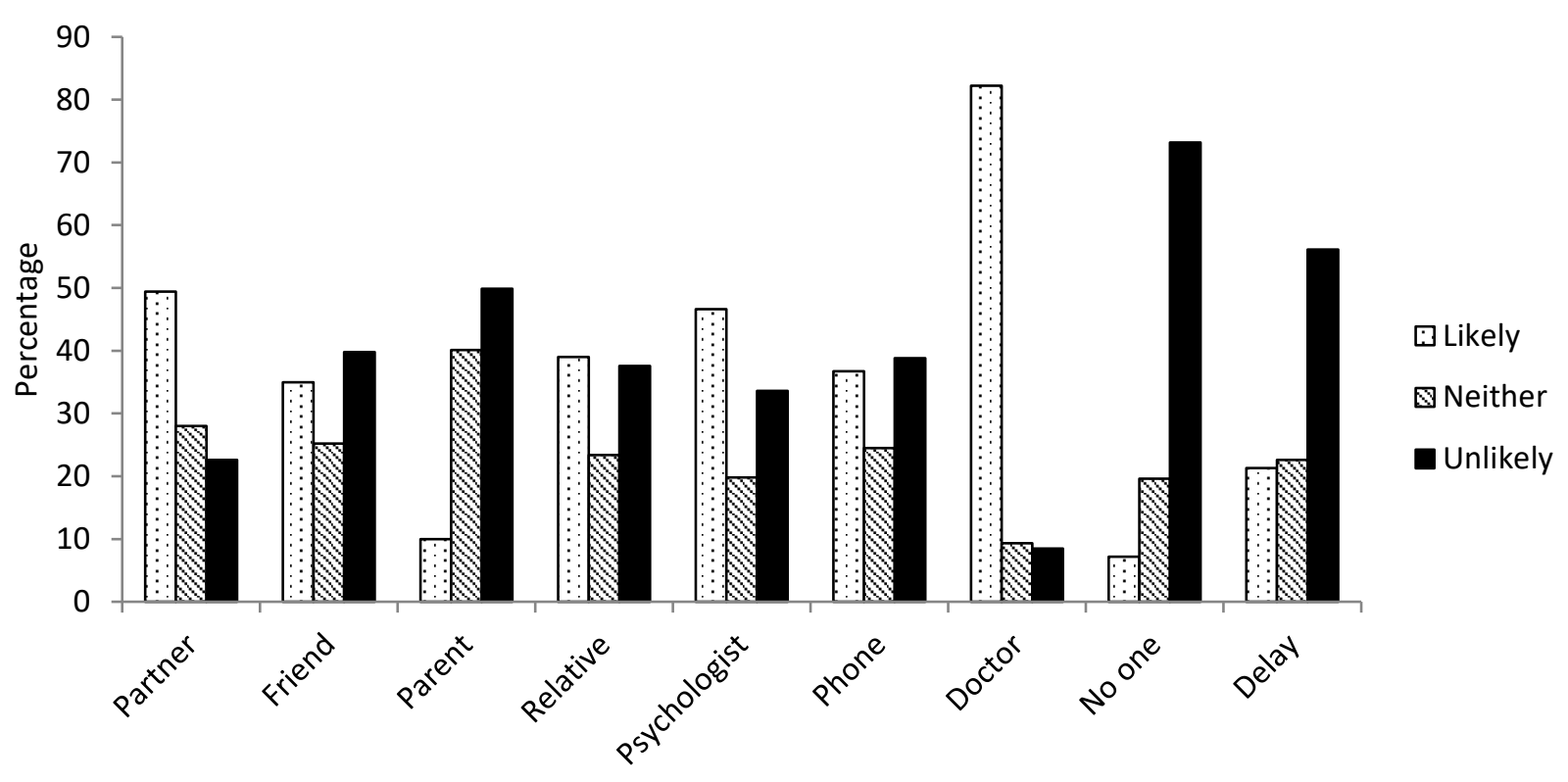

Figure 2. Help seeking intentions for 'self'. 


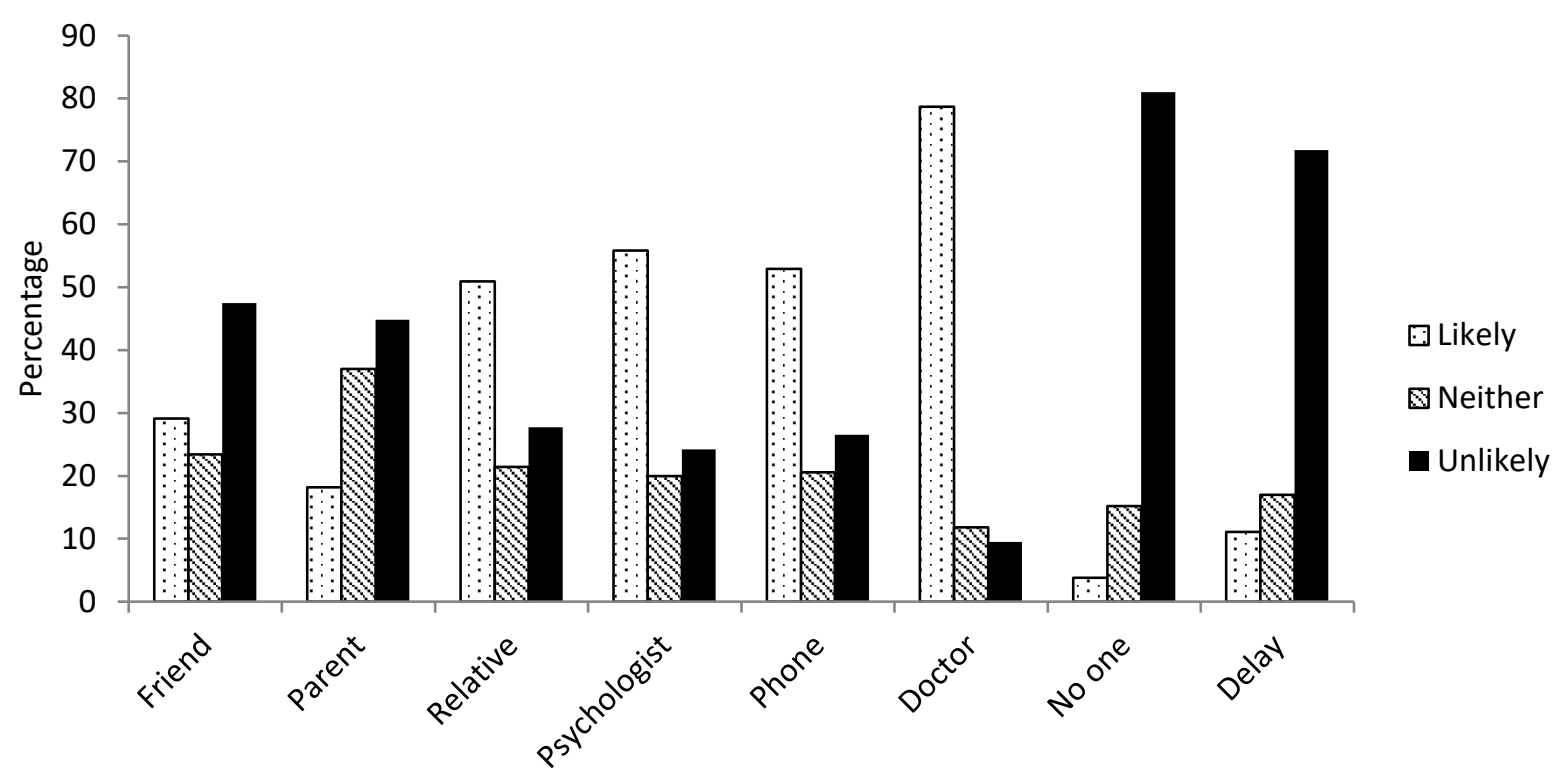

Figure 3. 'Proxy' help seeking intentions. 